\title{
Relative validity of two food-frequency questionnaires for children compared with 4-day diet diaries
}

\author{
L. C. A. Craig ${ }^{1,2}$, G. McNeill ${ }^{1,2}$, L. F. Masson ${ }^{1}$, J. Macdiarmid ${ }^{2}$, B. Holmes ${ }^{3}$, M. Nelson ${ }^{3}$ \\ and C. Sheehy ${ }^{4}$ \\ ${ }^{1}$ Section of Population Health, University of Aberdeen, Aberdeen, AB25 2ZD, UK, ${ }^{2}$ Rowett Institute of Nutrition and Health, \\ University of Aberdeen, Aberdeen, AB21 9SB, UK, ${ }^{3}$ King's College London, London, SE1 9NH, UK and ${ }^{4}$ Scottish Centre for \\ Social Research, Edinburgh, EH3 9AW, UK
}

The aim of the present study is to assess the relative validity of a parent-completed 140-item semi-quantitative FFQ for children aged 3-11 years (Scottish Collaborative Group (SCG) version C2) and a self-completed 146-item FFQ for children aged 12-16 years (SCG version C3) compared with 4-day non-weighed-diet diaries. Children who took part in the study were a sub-sample from the nation-wide Survey of sugar intake among children in Scotland ${ }^{(1)}$. One hundred and fifty eight children completed both an FFQ and a 4-day diary. Nutrient intakes were calculated using the NDNS databank. FFQ with energy intakes below the 2.5 and above the 97.5 centiles were excluded from the analysis to remove outliers (six subjects). Nutrient intakes were energy adjusted and relative agreement was assessed using Spearman rank correlation coefficients and cross-classification of the percentage of subjects in the same and opposite thirds of intake.

\begin{tabular}{|c|c|c|c|c|c|c|c|c|c|}
\hline & \multicolumn{4}{|c|}{ Intake } & \multirow[b]{3}{*}{$P$ for difference } & \multirow{2}{*}{\multicolumn{2}{|c|}{ Spearman rank correlation }} & \multirow{2}{*}{\multicolumn{2}{|c|}{ Percentage agreement }} \\
\hline & \multicolumn{2}{|c|}{ FFQ } & \multicolumn{2}{|c|}{ Diet diary } & & & & & \\
\hline & Median & IQR & Median & IQR & & $r_{s}$ & $P$ & Same thirds & Opposite thirds \\
\hline \multicolumn{10}{|l|}{$\overline{\text { Version C2 (n 96) }}$} \\
\hline Energy (MJ/d) & 7.32 & $6.33-8.98$ & 6.24 & $5.42-7.24$ & $<0.001$ & 0.21 & 0.037 & 45.8 & 16.7 \\
\hline Fat $\%$ energy & 32 & $30-35$ & 34 & $30-37$ & 0.011 & 0.46 & $<0.001$ & 43.8 & 10.4 \\
\hline SFA \% energy & 13 & $12-15$ & 14 & $13-16$ & 0.009 & 0.52 & $<0.001$ & 51 & 9.4 \\
\hline NMES \% energy & 15 & $13-19$ & 14 & $11-19$ & 0.063 & 0.36 & $<0.001$ & 39.6 & 12.5 \\
\hline $\operatorname{NSP}(g / d)$ & 13 & $10-16$ & 10 & $8-11$ & $<0.001$ & 0.55 & $<0.001$ & 58.3 & 12.5 \\
\hline Vitamin D $(\mu \mathrm{g} / \mathrm{d})$ & 1.3 & $0.9-2.0$ & 1.6 & $1.1-2.2$ & 0.162 & 0.29 & 0.004 & 38.5 & 11.5 \\
\hline Vitamin C (mg/d) & 151 & $111-215$ & 80 & $49-129$ & $<0.001$ & 0.32 & 0.001 & 41.7 & 12.5 \\
\hline Vitamin E (mg/d) & 7.4 & $5.8-8.9$ & 6.8 & $5.4-7.9$ & 0.002 & 0.29 & 0.005 & 41.7 & 14.6 \\
\hline Vitamin $A(\mu \mathrm{g} / \mathrm{d})$ & 600 & $467-803$ & 477 & $335-606$ & $<0.001$ & 0.45 & $<0.001$ & 46.9 & 9.4 \\
\hline $\mathrm{Fe}(\mathrm{mg} / \mathrm{d})$ & 9.6 & $7.7-11.0$ & 7.7 & $6.5-9.4$ & $<0.001$ & 0.48 & $<0.001$ & 53.1 & 9.4 \\
\hline $\mathrm{Ca}(\mathrm{mg} / \mathrm{d})$ & 1009 & $800-1268$ & 841 & $684-983$ & $<0.001$ & 0.47 & $<0.001$ & 52.1 & 6.3 \\
\hline Folate $(\mu \mathrm{g} / \mathrm{d})$ & 257 & $224-313$ & 174 & 144-206 & $<0.001$ & 0.56 & $<0.001$ & 51 & 7.3 \\
\hline \multicolumn{10}{|l|}{ Version C3 (n 56) } \\
\hline Energy (MJ/d) & 7.63 & $6.12-10.34$ & 7.29 & $6.17-9.17$ & 0.357 & 0.12 & 0.397 & 44.6 & 12.5 \\
\hline Fat $\%$ energy & 34 & $32-37$ & 33 & $31-37$ & 0.304 & 0.2 & 0.131 & 44.6 & 12.5 \\
\hline SFA \% energy & 15 & $13-16$ & 14 & $12-16$ & 0.186 & 0.35 & 0.008 & 48.2 & 8.9 \\
\hline NMES \% energy & 18 & $14-21$ & 16 & $11-21$ & 0.401 & 0.34 & 0.011 & 39.3 & 10.7 \\
\hline $\operatorname{NSP}(g / d)$ & 12 & $9-17$ & 11 & $9-13$ & 0.005 & 0.45 & $<0.001$ & 46.4 & 10.7 \\
\hline Vitamin $\mathrm{D}(\mu \mathrm{g} / \mathrm{d})$ & 1.3 & $0.8-2.3$ & 1.6 & $1.2-2.2$ & 0.014 & 0.27 & 0.046 & 42.9 & 14.3 \\
\hline Vitamin C (mg/d) & 123 & $91-192$ & 98 & $53-153$ & 0.01 & 0.19 & 0.16 & 42.9 & 14.3 \\
\hline Vitamin E (mg/d) & 7.5 & $5.7-10.8$ & 7.4 & $5.5-10.0$ & 0.285 & 0.24 & 0.074 & 39.3 & 10.7 \\
\hline Vitamin $A(\mu \mathrm{g} / \mathrm{d})$ & 580 & $416-939$ & 515 & $362-725$ & 0.075 & 0.32 & 0.015 & 39.3 & 14.3 \\
\hline $\mathrm{Fe}(\mathrm{mg} / \mathrm{d})$ & 9.1 & $6.8-12.8$ & 9.4 & $7.6-11.9$ & 0.757 & 0.3 & 0.025 & 41.1 & 12.5 \\
\hline $\mathrm{Ca}(\mathrm{mg} / \mathrm{d})$ & 1039 & $710-1402$ & 925 & $653-1189$ & 0.195 & 0.3 & 0.025 & 46.4 & 14.3 \\
\hline Folate $(\mu \mathrm{g} / \mathrm{d})$ & 255 & $174-328$ & 191 & $154-241$ & 0.001 & 0.42 & 0.001 & 51.8 & 8.9 \\
\hline
\end{tabular}

IQR, interquartile range.

Differences between the FFQ and diary for absolute nutrient intakes tended to be larger for version C2 than C3. However, for C2 Spearman rank correlation coefficients were significant $(P<0.05)$ for all nutrients but for $\mathrm{C} 3$ correlation coefficients were significant for all nutrients except energy, total fat (\% energy) and vitamins $\mathrm{C}$ and $\mathrm{E}$. The percentage of subjects classified in the same thirds was $>50$ for SFA (\% energy), NSP, Fe, Ca and folate for C2 and folate for C3. The percentage of subjects classified in opposite thirds was < 10 for SFA (\% energy), vitamin A, Fe, Ca and folate for C2 and SFA (\% energy) and folate for C3. Although the ranking agreement was better in younger children, absolute intakes agreed better between the two methods for older children.

The study was funded by the Food Standards Agency Scotland.

1. Sheehy C, McNeill G, Masson L et al. (2008) Survey of sugar intake among children in Scotland. Food Standards Agency Scotland. http:// www.food.gov.uk/scotland/scotnut/scotsug 